\title{
Pinnularia (Bacillariophyta) do curso inferior do rio Negro, Amazonas, Brasil: taxonomia e distribuição temporal*
}

\begin{abstract}
Andreia Cavalcante PEREIRA², Lezilda Carvalho TORGAN², Sérgio MELO
RESUMO

Este estudo relata a taxonomia e distribuição temporal do gênero Pinnularia ocorrente no curso inferior do rio Negro $\left(03^{\circ} 02^{\prime} 46,5^{\prime \prime}\right.$ e e $\left.60^{\circ} 15^{\prime} 13,1^{\prime \prime} \mathrm{W}\right)$ ao longo de um ciclo anual. O trabalho foi conduzido a partir da análise de amostras coletadas na coluna d'água, em escala mensal, entre os meses de outubro de 2002 a setembro de 2003. As espécies foram descritas e comentadas com base na sua morfologia e morfometria. Onze espécies e quatro variedades foram identificadas, ilustradas e incluídas em chave taxonômica. Dois táxons registrados neste estudo, P. sterrenburgii var. sterrenburgii Metzeltin \& Lange-Bertalot e P. subgibba var. capitata Metzeltin \& Krammer, constituem primeira citaçáo de ocorrência para o rio Negro. Temporalmente, a maior riqueza de espécies ocorreu entre os meses de outubro a dezembro de 2002, período de águas baixas, quando houve provavelmente maior interação entre água e sedimento possibilitando aporte de indivíduos da região bentônica. Considerando a ocorrência dos táxons ao longo do estudo, somente $P$. confirma foi considerada frequente, estando presente em mais de $50 \%$ das amostras analisadas.
\end{abstract}

PALAVRAS-CHAVE: águas pretas, diatomáceas, plâncton, região amazônica

\section{Pinnularia (Bacillariophyta) from the lower course of Negro river (Amazon, Brazil): taxonomy and temporal distribution}

\section{ABSTRACT}

This study reports the taxonomy and temporal distribution of the Pinnularia genus occurring in the lower course Negro river $\left(03^{\circ} 02^{\prime} 46,5^{\prime}\right.$ S e $\left.60^{\circ} 15^{\prime} 13,1^{\prime} \mathrm{W}\right)$ along an annual cycle. Samples were collected in the water column monthly, from October 2002 to September 2003. The species were described and commented based on their morphology and morphometry. Eleven species and four varieties are registered, illustrated and incorporated in a taxonomic key. P. sterrenburgii var. sterrenburgii Metzeltin \& Lange-Bertalot and P. subgibba var. capitata Metzeltin \& Krammer are the first references to Negro river. Temporally, the highest species richness occurred from October and December 2002, low water period, when happened more interaction between water and sediment, and the contribution of the benthos individuals. On base in taxa occurrence during this study, only $P$. confirma was frequently, with occurrence in more than $50 \%$ of sample analyzed.

KEYWORDS: black water, diatoms, plankton, Amazonian region

\footnotetext{
* Parte da tese de doutorado da primeira autora

1 Universidade Federal do Rio Grande do Sul. Programa de Pós-Graduação em Botânica. E-mail: diatomaceas@gmail.com

2 Fundação Zoobotânica do Rio Grande do Sul - Rua Salvador França, 1427, CEP 9060-000, Porto Alegre, RS. E-mail: lezilda-torgan@fzb.rs.gov.br.

${ }^{3}$ Universidade Federal do Oeste do Pará - Instituto de Ciências e Tecnologia das Águas. Av. Vera Paz, s/n - Bairro Salé, CEP: 68.035-110, Santarém, PA. E-mail: melo.joaopedro@gmail.com
} 


\section{INTRODUÇÃO}

Pinnularia é um gênero de diatomáceas que ocorre predominantemente em águas continentais, oligotróficas, com baixa concentraçáa de eletrólitos e baixos valores de pH (Uherkovich 1984; Metzeltin e Lange-Bertalot 1998; Krammer 2000). As águas interiores amazônicas, pelo fato de apresentarem as referidas características, possuem condiçôes favoráveis ao desenvolvimento deste táxon.

A bacia do rio Negro cobre $700.000 \mathrm{~km}^{2}$ e compreende mais de $10 \%$ da área total da bacia Amazônica (Goulding et al. 2003). As suas águas possuem coloraçâo marromavermelhada, originada das altas quantidades de substâncias fúlvicas e húmicas, baixos valores de $\mathrm{pH}$ e de condutividade elétrica (Sioli 1984).

Estudos sobre algas do rio Negro e afluentes que apresentam descrições e/ou comentários taxonômicos sobre Pinnularia são escassos. O primeiro foi redigido por Uherkovich (1976) em que dentre as 33 espécies de diatomáceas listadas, somente a espécie Pinnularia interrupta W. Smith foi citada. Uherkovich e Rai (1979), mencionaram sete espécies [Pinnularia braunii (Grunow) Cleve, P. cardinalis (Ehrenberg) Smith, P. gibba Ehrenberg var. gibba f. subundulata Mayer, $P$. maior (Kützing) Cleve, P. polyonca (Brébisson) Müller, P. subcapitata Gregory var. hilseana (Janish) Müller, P. legumen Ehrenberg] para o fitoplâncton do rio Carabinani, localizado na bacia do rio Negro.

Em amostras do epilíton de um ponto do baixo rio Negro, Fukushima e Xavier (1988) apresentaram duas espécies [P. subcapitata Gregory; P. hemiptera (Kutzing) Rabenhost]. No perifíton do rio Jaú, Diaz-Castro et al. (2003), descreveram 12 espécies, das quais nove foram identificadas [Pinnularia acrosphaeria Smith, P. braunii (Grunow) Cleve var. amphicephala (Mayer) Hustedt, P. graciloides Hustedt, P. hemiptera (Kutzing) Rabenhost, P. hemiptera (Kutzing) Rabenhost var. interrupta Cleve, P. interrupta Smith var. biceps (Gregory) Cleve, P. maior var. transversa (Smith) Cleve, P. rostratissima Hustedt, P. streptoraphe Cleve var. subacuta Frenguelli]. Mais recentemente, Metzeltin e Lange-Bertalot (1998, 2007) descreveram sete novas espécies (P. aquanigrae, P. boyeriformis, P. microflamma, P. mollenhaueri, P. perinstabilis, P. permontana, P. salomoniae) para o rio Negro e Wetzel (2011) apresenta 63 táxons específicos e infra-específicos encontrados no plâncton e perifíton ao longo do rio Negro, no período de enchente (fevereiro a março de 2005).

Apesar da contribuição científica sobre o gênero Pinnularia em águas pretas da Amazônia, em especial, na bacia do rio Negro, a grande parte destas carece de chave, descriçóes e suas variaçôes em relação aos períodos hidrológicos. $\mathrm{O}$ presente estudo vem, portanto, descrever e ilustrar as espécies de Pinnularia encontradas no curso inferior do rio Negro e verificar sua distribuição ao longo de um ciclo anual. Abordam-se também as variaçôes morfométricas e a distribuição dos táxons na região amazônica.

\section{MATERIAL E MÉTODOS}

As amostragens no rio Negro foram realizadas mensalmente (exceto mês de junho) em um ponto localizado cerca de $30 \mathrm{~km}$ a noroeste da cidade de Manaus (03002'46,5"S e $60^{\circ} 15^{\prime} 13,1$ 'W), no período de outubro de 2002 a setembro de 2003. As amostras foram obtidas com rede de plâncton (abertura de malha de $25 \mu \mathrm{m}$ ) através de arrastos verticais e horizontais na região central do rio, sendo fixadas com solução de Transeau (Bicudo e Menezes 2006). No laboratório, subamostras de $10 \mathrm{ml}$ foram oxidadas segundo o método de Stosch (1970) para a montagem das lâminas permanentes. Utilizou-se a resina Naphrax como meio de inclusão (Índice de Refração = 1,74). O material foi analisado em microscópio óptico, marca Leica, equipado com câmera fotográfica digital Leica DFC 290.

A identificação das espécies foi baseada nas características morfológicas e morfométricas das valvas utilizando as obras de Schmidt et al. (1874-1959), Hustedt (1965), Patrick e Reimer (1966), Simonsen (1987), Krammer (2000), Metzeltin e Lange-Bertalot $(1998,2002,2007)$. Na classificação, para as categorias de divisão a classe seguiu-se o sistema de Medlin e Kaczmarska (2004) e de ordem a família o sistema de Round et al.(1990). As amostras foram depositadas no Herbário do Instituto Nacional de Pesquisas da Amazônia (INPA). Os números de registros das amostras, bem como o período de amostragem e demais informaçôes sobre algumas condiçốes físico-química da água são apresentados na Tabela 1.

Com relação à freqüência das espécies, foram considerados freqüentes (F) as que tiveram presença acima de $50 \%$ nos meses observados; esporádicas (E) e raras (R) as que estavam presentes acima e abaixo de $20 \%$, respectivamente.

Durante as coletas foram efetuadas medidas de temperatura, $\mathrm{pH}$ e condutividade elétrica, através de leitura direta, com potenciômetro portátil (Yellow Springs Instruments, modelo 63, Ohio, Estados Unidos). A transparência da água foi medida pela profundidade de extinçấo do disco de Secchi na coluna d'água e, os valores de níveis da água do rio Negro foram obtidos no porto da cidade de Manaus.

\section{RESULTADOS E DISCUSSÃO}

\section{Taxonomia}

Os táxons pertencem à classe Bacillariophyceae Medlin e Kaczmarska, ordem Naviculales Bessey, família Pinnulariaceae Mann. A chave a seguir apresenta as características morfológicas diacríticas capazes de separar os táxons identificados. 


\section{Chave para identificação dos táxons de Pinnularia.}

1. Presença de fascia

2. Valvas lineares

3. Sternum da rafe estreito

4. Área central com fascia estreita

5. Margens valvares

paralelas a levemente

onduladas

5. Margens valvares infladas na região mediana

4. Área central com fascia larga

6. Extremidades largas, cuneado-arredondadas

6. Extremidades capitadoarredondadas

3. Sternum da rafe largo

7. Margens valvares trionduladas

8. Área central com fascia larga

8. Área central com fascia estreita

7.Margens valvares não trionduladas

9. Extremidades cuneadoarredondadas

9. Extremidades capitadocuneadas

1. P. aquaenigrae

2. P. boyeriformis

5. P. meridiana var. concava

11. $P$.

rhombofasciata

10. P. perumbrosa

15. P. subgibba var. capitata

\section{4. $P$.}

instabiliformis

14. P. subboyeri

2. Valvas com outras formas

10. Valvas linear-elíptica com estrias paralelas

10. Valvas lanceoladas com estrias radiadas, convergentes em direção às extremidades

1. Ausência de fascia

11. Valvas linear-elípticas

12. Estrias paralelas

12. Estrias radiadas, convergentes em direção às extremidades

11. Valvas lineares

13. Margens valvares paralelas

14. Extremidades arredondadas

14. Extremidades cuneado-obtusa

13. Margens valvares infladas na região mediana

12. $P$. rostratissima var. ventricosa

\section{P. mollenhaueri}

9. P. permontana
3. P. confirma

8. P. perinstabilis

13. P. sterrenburgii var. sterrenburgii

1. Pinnularia aquaenigrae Metzeltin e Lange-Bertalot, Iconographia Diatomologica, vol. 18. 2007. (Figura 1A).

Valvas lineares com margens paralelas a levemente onduladas, extremidades arredondadas. Comprimento 76-82,5 $\mu \mathrm{m}$, largura 13,3-14 $\mu \mathrm{m}$. Sternum da rafe estreito alcançando aproximadamente $1 / 4$ da largura da valva, área central com fascia estreita. Rafe lateral com extremidades proximais fletidas. Estrias $(10-11 / 10 \mu \mathrm{m})$ levemente radiadas e convergentes em direção às extremidades valvares.

Comentários: Quando comparado com as ilustraçôes do material tipo, originado do rio Negro, efetuadas por Metzeltin e Lange-Bertalot (2007), os espécimes analisados apresentaram valvas levemente onduladas similares às apresentadas no material tipo, embora na diagnose da espécie tal característica não tenha sido mencionada. Recentemente foi registrada novamente no rio Negro por Wetzel (2011).

Material examinado: INPA 223980, 223987, 223998.

\section{Pinnularia boyeriformis Krammer e Metzeltin, Iconographia} Diatomologica, vol 5. 1998. (Figura 1B).

Valvas lineares com margens infladas na região mediana extremidades arredondadas. Comprimento $100 \mu \mathrm{m}$, largura $16 \mu \mathrm{m}$. Sternum da rafe estreito, área central com fascia estreita, assimétrica, alcançando as margens. Rafe lateral com extremidades proximais fletidas. Estrias $(9-10 / 10 \mu \mathrm{m})$ radiadas na região mediana, a convergentes em direção às extremidades valvares.

Material examinado: INPA 223985, 223987, 223992.

Comentários: A espécie registrada neste estudo assemelhase morfologicamente com as ilustraçóes do material tipo realizadas por Metzeltin e Lange-Bertalot (1998). Recentemente esta espécie foi encontrada no plâncton do rio Negro por Wetzel (2011).

\section{Pinnularia confirma Metzeltin e Krammer, Iconographia Diatomologica, vol. 5. 1998. (Figura 1C).}

Valvas lineares com margens paralelas, extremidades arredondadas. Comprimento 46-57 $\mu \mathrm{m}$, largura 7,5-8,0 $\mu \mathrm{m}$. Sternum da rafe largo alcançando aproximadamente $1 / 2$ da largura da valva, ausência de área central. Rafe lateral com extremidades proximais fletidas. Estrias $(13-14 / 10 \mu \mathrm{m})$ paralelas ao longo das valvas.

Comentários: esta espécie foi primeiramente encontrada nas Guyanas (Metzeltin e Lange-Bertalot 1998) e recentemente tanto no plâncton do lago Tupé (Pereira 2009) como no perifíton do rio Negro (Wetzel 2011).

Material examinado: INPA 223980, 223985, 223987 223992, 224000, 224005, 224017. 

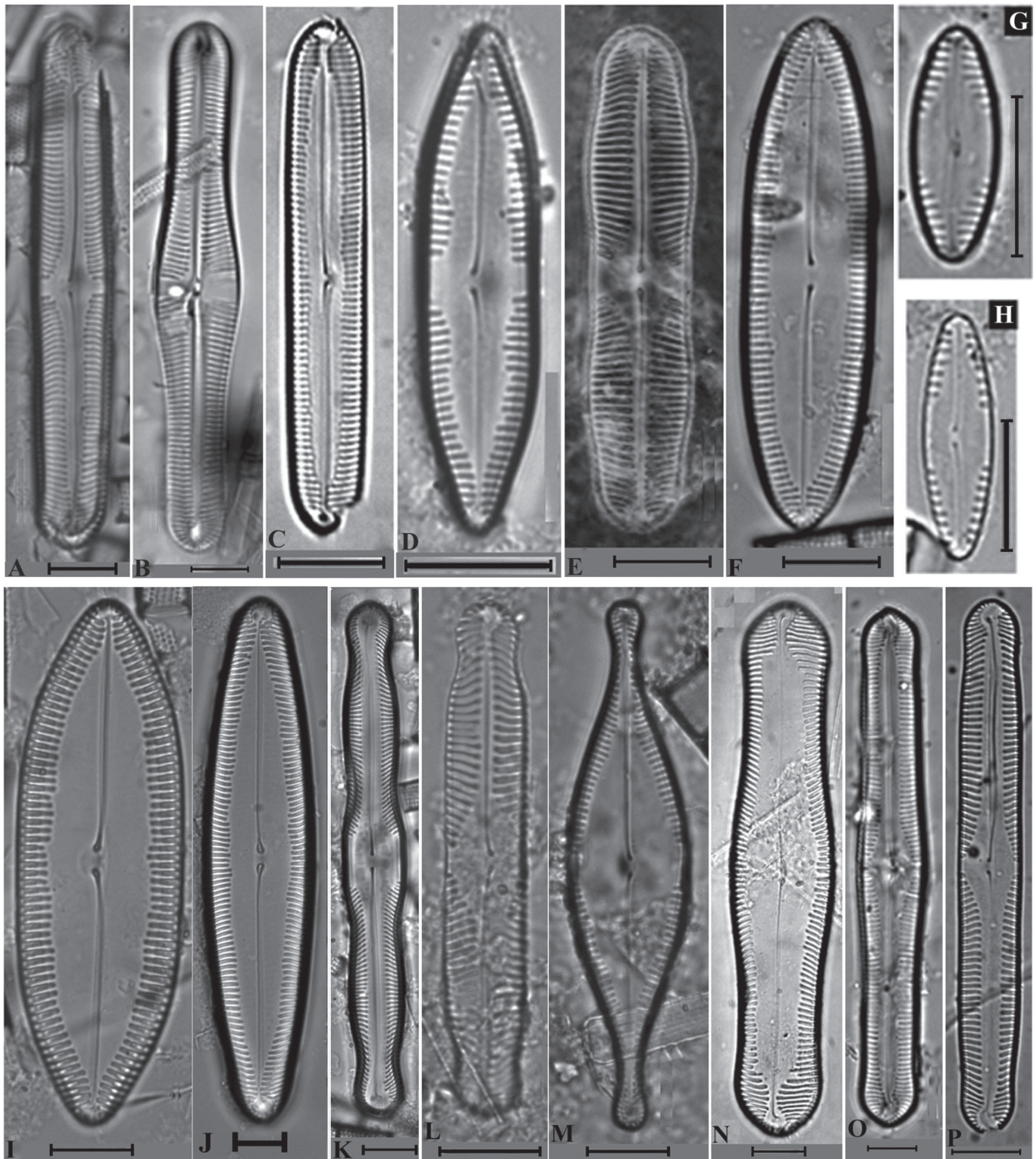

Figura 1 - Representação fotográfica das espécies do gênero Pinnularia encontradas no curso inferior do rio Negro - AM. A- Pinnularia aquaenigrae; B- $P$. boyeriformis; C- P. confirma; D- P. instabiliformis; E- P. meridiana var. concava; F- P. mollenhaueri; G,H- P. pogoii; I- P. perinstabilis; J- P. permontana; K- $P$. perumbrosa; L- P. rhombofasciata; M- P. rostratissima var. ventricosa; N- $P$. sterrenburgii var. sterrenburgii; 0 - $P$. subboyeri; $P$ - $P$. subgibba var. capitata. Escala: $10 \mu \mathrm{m}$. 


\section{Pinnularia instabiliformis Krammer e Metzeltin, Iconographia} Diatomologica, vol. 5. 1998. (Figura 1D).

Valvas lineares com margens paralelas, extremidades cuneado-arredondadas. Comprimento 30-38,3 $\mu \mathrm{m}$, largura 9-10 $\mu \mathrm{m}$. Sternum da rafe largo, alcançando $1 / 2$ da largura da valva, área central com fascia larga, alcançando as margens. Rafe lateral com extremidades proximais fletidas. Estrias (13$14 / 10 \mu \mathrm{m})$ paralelas ao longo das valvas.

Comentários: o material examinado apresenta pequena diferença do material tipo estudado por Metzeltin e LangeBertalot (1998), oriundo de sedimentos fósseis (11.000 AP) do lago Calado no estado do Amazonas, por apresentar dimensôes ligeiramente menores e por possuir maior número de estrias em $10 \mu \mathrm{m}$. No entanto, apenas estas diferenças não são o suficiente para a proposição de um novo táxon. Sugerem-se, assim estudos com maior número de indivíduos a fim de verificar se há uma variabilidade morfológica no grupo que permite alterar o atual táxon. Esta espécie foi registrada recentemente no plâncton do lago Tupé com características morfológicas similares as da espécie tipo (Pereira 2009) e no perifíton do rio Negro (Wetzel 2011). Este último registro, no entanto, não permite a confirmação da espécie devido à ausência de ilustração.

Material examinado: INPA 223980, 223987, 223992, 224017.

5. Pinnularia meridiana var. concava Metzeltin e Krammer, Iconographia Diatomologica, vol. 5. 1998. (Figura 1E).

Valvas lineares com margens tri-onduladas, extremidades largas cuneado-arredondadas. Comprimento 56-57 $\mu \mathrm{m}$, largura 11-12 $\mu \mathrm{m}$. Sternum da rafe estreito alcançando aproximadamente $1 / 4$ da largura das valvas, área central com fascia larga, alcançando as margens. Rafe lateral com extremidades proximais fletidas entre si. Estrias $(11-12 / 10 \mu \mathrm{m})$ radiadas na regiáo mediana e convergentes nas extremidades valvares.

Comentários: os exemplares observados apresentaram menor comprimento e maior densidade de estrias do que o material encontrado em sedimento fóssil de $11.000 \mathrm{AP}$ oriundo da regiāo Amazônica (61-63 $\mu \mathrm{m}$ de compr. 10 estrias em $10 \mu \mathrm{m}$ ) analisado por Metzeltin e Lange-Bertalot (1998).

Material examinado: INPA 223985, 223987.

6. Pinnularia mollenhaueri Metzeltin e Lange-Bertalot, Iconographia Diatomologica, vol. 18. 2007. (Figura 1F).

Valvas linear-elipticas com margens paralelas, extremidades cuneado-arredondadas. Comprimento $58 \mu \mathrm{m}$, largura 14 $\mu \mathrm{m}$. Sternum da rafe largo alcançando $1 / 2$ da largura da valva, ausência de área central. Rafe lateral com extremidades proximais fletidas. Estrias $(10-11 / 10 \mu \mathrm{m})$ curtas e paralelas ao longo das valvas e próximas da rafe nas extremidades valvares.
Material examinado: INPA 223980.

Comentários: Morfologicamente, o exemplar documentado neste estudo apresentou-se similar ao material tipo, originário do rio Negro, ilustrado por Metzeltin e LangeBertalot (2007). Recentemente foi registrado no lago Tupé (Pereira 2009) e no do rio Negro (Wetzel 2011).

\section{Pinnularia perinstabilis Metzeltin e Krammer, Iconographia Diatomologica, vol. 5. 1998. (Figura 1I).}

Valvas lineares com margens paralelas, extremidades cuneado-obtusa. Comprimento 68 - $70 \mu \mathrm{m}$, largura 22,2$24 \mu \mathrm{m}$. Sternum da rafe largo alcançando $1 / 2$ da largura da valva, ausência de área central. Rafe lateral com extremidades proximais fletidas. Estrias $(8-9 / 10 \mu \mathrm{m})$ paralelas, levemente convergentes nas extremidades valvares.

\section{Material examinado: INPA 223980.}

Comentários: Esta espécie foi encontrada na região amazônica em sedimento fóssil do rio Arapiuns e do rio Negro (Metzeltin e Lange-Bertalot, 1998, 2007), no perifíton do rio Negro (Wetzel 2011) e no plâncton do lago Tupé (Pereira 2009).

\section{Pinnularia permontana Krammer e Metzeltin, Iconographia Diatomologica, vol. 5. 1998. (Figura 1J).}

Valvas linear-elipticas, extremidades levemente rostradas, arredondadas. Comprimento 118,5-120 $\mu \mathrm{m}$, largura 20-23,7 $\mu \mathrm{m}$. Sternum da rafe largo alcançando $1 / 2 \mathrm{da}$ largura da valva, ausência de área central. Rafe lateral com extremidades proximais fletidas. Estrias (10-11/10 $\mu \mathrm{m})$ radiadas, convergentes em direção às extremidades valvares. Contudo, estes foram caracteres considerados insuficientes para a proposição de uma variedade e sugere-se uma analise populacional para averiguar melhor estes caracteres.

Comentários: os exemplares observados diferem do material tipo, oriundo do rio Negro, regiáo amazônica, descrito por Metzeltin e Lange-Bertalot (1998), por possuírem menores dimensóes e valor ligeiramente superior no que se refere ao número de estrias em $10 \mu \mathrm{m}$. Contudo, estes caracteres foram considerados insuficientes para a proposição de uma variedade. Porém, sugere uma analise populacional para averiguar melhor estes caracteres.

Material examinado: INPA 223985, 223987.

9. Pinnularia perumbrosa Metzeltin e Krammer, Iconographia Diatomologica, vol. 18. 2007. (Figura 1K).

Valvas lineares com margens fortemente tri-onduladas, infladas na região mediana, extremidades capitado-cuneadas arredondadas. Comprimento 100-111 $\mu \mathrm{m}$, largura $12 \mu \mathrm{m}$. Sternum da rafe largo alcançando $1 / 3$ da largura da valva, área central com fascia larga, alcançando as margens valvares. Rafe lateral com extremidades proximais fletidas. Estrias 
$(11 / 10 \mu \mathrm{m})$ radiadas, convergentes em direção às extremidades valvares.

Comentários: esta espécie foi descrita inicialmente em Metzeltin e Lange-Bertalot (1998) como P. umbrosa Sovereign var. tropica Metzeltin e Krammer para a regiâo Amazônica, em sedimento fóssil de 11.000 AP. Posteriormente, Metzeltin e Lange-Bertalot (2007) reavaliando as características morfométricas de $P$. umbrosa Sovereign consideraram o táxon em questáo distinto, denominando-o de $P$. perumbrosa, já que o epíteto tropica pertencia a outra espécie (Pinnularia tropica Hustedt). O material analisado no presente estudo confere com as dimensóes e/ou no número de estrias dos exemplares observados por Metzeltin e Lange-Bertalot (2007) oriundos do sedimento do lago Calado. Recentemente, esta espécie foi registrada para o rio Negro por Wetzel (2011).

Material examinado: INPA 223980, 223985, 223987

10. Pinnularia pogoii Sherer, Diatom Research, vol.3, n.1. 1988. (Figura 1G, H).

Valvas linear-elípticas, extremidades cuneadoarredondadas. Comprimento 16-19,8 $\mu \mathrm{m}$ largura 5-5,8 $\mu \mathrm{m}$. Sternum da rafe largo alargando-se em direção à área central, área central com fascia larga, alcançando as margens valvares. Rafe lateral com extremidades proximais fletidas. Estrias $(12 / 10 \mu \mathrm{m})$ curtas e paralelas ao longo das valvas.

Comentários: Esta espécie foi identificada primeiramente como $P$. oominensis (nome ilegítimo por não apresentar diagnose em latim) e citada para o lago Calado e Jucuruí por Metzeltin e Lange-Bertalot $(1998 ; 2007)$ e lago Tupé por Pereira (2009), como P. oominensis. Recentemente foi registrada com o nome legítimo $P$. pogoii para o rio Negro por Wetzel (2011).

Material examinado: INPA 223987, 223992, 224000.

11. Pinnularia rhombofasciata Krammer e Metzeltin, Iconographia Diatomologica, vol. 5. 1998. (Figura 1L).

Valvas lineares com margens paralelas, levemente onduladas, extremidades capitado-arredondadas. Comprimento 56,4-58 $\mu \mathrm{m}$, largura 8,3- $9 \mu \mathrm{m}$. Sternum da rafe estreito, área central com fascia larga e assimétrica, alcançando as margens. Rafe lateral com extremidades proximais fletidas. Estrias (9-10/10 $\mu \mathrm{m})$ radiadas, convergente em direção às extremidades valvares.

Comentários: os exemplares analisados apresentaram similares aos da descrição original (Metzeltin e Lange-Bertalot, 1998), porém com dimensōes menores das encontradas por Wetzel (2011) no fitoplâncton do curso inferior do rio Negro.

Material examinado: INPA 223987.
12. Pinnularia rostratissima var. ventricosa Metzeltin e LangeBertalot, Iconographia Diatomologica, vol. 5. 1998. (Figura 1M).

Valvas largamente lanceoladas com margens convexas, fortemente infladas na regiáo mediana, extremidades capitadoarredondadas. Comprimento 55,8-68 $\mu \mathrm{m}$, largura 14,6 - 15,3 $\mu \mathrm{m}$. Sternum da rafe largo alcançando mais que a metade da largura da valva, área central com fascia larga, alcançando as margens valvares. Rafe lateral com extremidades proximais fletidas. Estrias $(11-12 / 10 \mu \mathrm{m})$ radiadas na regiáo mediana, levemente convergentes em direção as extremidades valvares.

Material examinado: INPA 223980, 223985, 223987, 223994.

Comentários: os exemplares analisados, quando comparado com as ilustraçôes da variedade tipo, apresentaram similares aos da descrição original (Metzeltin e Lange-Bertalot 1998). Esta espécie foi encontrada no estado do Amazonas (Metzeltin e Lange-Bertalot 1998), rio Negro (Metzeltin e Lange-Bertalot 2007; Wetzel 2011) e lago Tupé (Pereira 2009). Esta variedade assemelha-se a $P$. braunii registrada no rio Carabinani, na bacia do rio Negro, por Uherkovich e Rai (1979), porém apresenta dimensôes e número de estrias menores.

\section{Pinnularia sterrenburgii var. sterrenburgii Metzeltin e} Lange-Bertalot, Iconographia Diatomologica, vol. 18. 2007. (Figura $1 \mathrm{~N})$.

Valvas lineares com margens fortemente infladas na região mediana, extremidades largas, subcapitado-cuneadas. Comprimento 101,6-109,8 $\mu \mathrm{m}$, largura 22,2-23 $\mu \mathrm{m}$. Sternum da rafe largo alcançando $1 \frac{1}{2}$ da largura da valva, ausência de área central. Rafe lateral com extremidades proximais fletidas. Estrias $(9-10 / 10 \mu \mathrm{m})$ radiadas, paralelas e prolongadas até próximo a rafe nas extremidades valvares.

Comentários: morfologicamente, o material assemelha-se ao material tipo oriundo de sedimento do lago Calado, regiáo amazônica, analisado por Metzeltin e Lange-Bertalot (2007), com exceção do número de estrias, apresentando valores maiores $(9-10 / 10 \mu \mathrm{m}$ ao invés de 7,5/10 $\mu \mathrm{m})$. O registro desta espécie constitui sua primeira citação de sua ocorrência na bacia do rio Negro.

Material examinado: INPA 223980, 223987, 224017.

14. Pinnularia subboyeri Metzeltin e Krammer. Iconographia Diatomologica, vol. 5. 1998. (Figura 10).

Valvas lineares com margens infladas na região mediana, extremidades levemente capitado-cuneadas. Comprimento $111 \mu \mathrm{m}$, largura 14,4 $\mu \mathrm{m}$. Sternum da rafe largo alcançando $1 / 3$ da largura da valva, área central com fascia estreita. Rafe lateral com extremidades proximais fletidas. Estrias (10/10 $\mu \mathrm{m})$ levemente radiadas, paralelas nas extremidades valvares.

Material examinado: INPA 223987. 
Comentários: Recentemente esta espécie foi encontrada no lago Tupé por Pereira (2009).

15. Pinnularia subgibba var. capitata Metzeltin e Krammer, Iconographia Diatomologica, vol. 5. 1998. (Figura 1P).

Valvas lineares com margens levemente tri-onduladas, extremidades levemente capitado-arredondadas. Comprimento 85-90 $\mu \mathrm{m}$, largura 10-10,6 $\mu \mathrm{m}$. Sternum da rafe largo alcançando $1 / 3$ da largura da valva, área central com fascia estreita. Rafe lateral com extremidades proximais fletidas. Estrias $(9-10 / 10 \mu \mathrm{m})$ radiadas, convergentes em direção as extremidades valvares.

Comentários: o material assemelha-se ao material tipo oriundo do lago Calado, regiáo amazônica (Metzeltin e LangeBertalot 1998). O registro desta espécie constitui sua primeira citação de ocorrência na bacia do rio Negro.

Material examinado: INPA 223980, 223987.

\section{Distribuição temporal}

As águas do curso inferior do rio Negro mostraram baixos valores de $\mathrm{pH}(3,7$ a 5,9) e de condutividade elétrica (7,9 a 35,9 $\left.\mu \mathrm{S} \mathrm{cm}^{-1}\right)$. Águas com características ácidas e de baixa condutividade elétrica são frequentes nos ecossistemas amazônicos de águas pretas, como já demonstrado em estudos realizados no rio Jaú (Díaz-Castro et al. 2003), no lago Tupé (Rai e Hill 1981) e no rio Negro próximo a Manaus (Junk e Silva 1995), entre outros. A transparência da água variou entre o máximo de $1,25 \mathrm{~m}$ em janeiro e julho de 2003 ao mínino $0,90 \mathrm{~m}$ em outubro de 2002 e maio de 2003 . O nível da água oscilou entre 18,5 m (águas baixas) e 28,1 m (águas altas), e a temperatura manteve-se acima de $28^{\circ} \mathrm{C}$ (Tabela 1 ).

Tabela 1 - Registro das amostras depositadas no Herbário do Instituto Nacional de Pesquisas da Amazônia (INPA), seus respectivos meses/ano de coletas e valores de temperatura, $\mathrm{pH}$, condutividade elétrica, transparência e nível da água no curso inferior do Rio Negro, no período de outubro de 2002 a setembro de 2003.

\begin{tabular}{|c|c|c|c|c|c|c|}
\hline $\begin{array}{l}\text { Amostra } \\
\text { INPA }\end{array}$ & Mês/Ano & $\begin{array}{c}\text { Temp. } \\
\left({ }^{\circ} \mathrm{C}\right)\end{array}$ & $\mathrm{pH}$ & $\begin{array}{c}\text { Cond. } \\
\text { Elétrica } \\
\left(\mu \mathrm{S} \mathrm{cm}^{-1}\right)\end{array}$ & $\begin{array}{l}\text { Transparência } \\
\text { Secchi (m) }\end{array}$ & $\begin{array}{c}\text { Nível } \\
\text { da água } \\
\text { (m) }\end{array}$ \\
\hline 223980 & Out/2002 & 31,8 & 4,3 & 12,0 & 0,90 & 20,5 \\
\hline 223985 & Nov/2002 & 31,9 & 4,3 & 11,0 & 0,95 & 18,5 \\
\hline 223987 & Dez/2002 & 32,0 & 4,6 & 7,90 & 1,00 & 20,6 \\
\hline 223992 & Jan/2003 & 30,3 & 4,5 & 10,0 & 1,25 & 22,3 \\
\hline 223994 & $\mathrm{Fev} / 2003$ & 30,1 & 5,1 & 10,4 & 1,20 & 22,3 \\
\hline 223998 & Mar/2003 & 30,0 & 5,0 & 12,0 & 1,10 & 23,0 \\
\hline 224000 & $\mathrm{Abr} / 2003$ & 30,0 & 5,9 & 13,5 & 1,10 & 24,7 \\
\hline 224005 & Mai/2003 & 29,0 & 3,7 & 35,9 & 0,90 & 26,7 \\
\hline 224010 & $\mathrm{Jul} / 2003$ & 29,7 & 3,8 & 14,9 & 1,20 & 28,1 \\
\hline 224012 & Ago/2003 & 29,5 & 3,7 & 11,1 & 1,25 & 26,9 \\
\hline 224017 & Set/2003 & 28,0 & 4,0 & 14,1 & 1,00 & 24,6 \\
\hline \multicolumn{2}{|c|}{ Mínimo } & 28 & 3,7 & 7,9 & 0,90 & 18,5 \\
\hline \multicolumn{2}{|c|}{ Máximo } & 32 & 5,9 & 35,9 & 1,25 & 28,1 \\
\hline
\end{tabular}

Em relação à distribuição temporal das espécies ao longo do período estudado, a maioria das espécies foi rara e esporádica, ou seja, estiveram presentes em menos de 50\% das amostras analisadas. Somente $P$. confirma foi frequente, com $64 \%$ de presença no período de estudo (Tabela 2). Esta espécie foi registrada recentemente em duas localidades da bacia do rio Negro, no perifíton do rio Negro (Wetzel 2011) e no plâncton do lago Tupé (Pereira 2009) onde também foi frequente ao longo dos estudos, indicando uma afinidade para as águas pretas da Amazônia caracterizadas por baixos valores de $\mathrm{pH}$ e baixos valores de condutividade elétrica.

Pinnularia é um gênero bentônico (Round et al. 1990), fato que torna pouco comum sua presença no plâncton. A variação da riqueza de espécies em relaçáo ao ciclo hidrológico do rio Negro pode confirmar essa tendência (Figura 2). Os valores maiores de riqueza (6 a 13 táxons) ocorreram no período de águas baixas (outubro a dezembro de 2002), em que o nível hidrométrico foi $<20,6 \mathrm{~m}$. Neste período é maior a interação entre o sedimento e a coluna de água, condiçóes que podem ter promovido o aporte das valvas de Pinnularia à coluna de água. No período de águas altas, observou-se apenas um táxon, chegando à inexistência no mês de julho e agosto de 2003.

A relação de riqueza de Pinnularia com o regime hidrológico encontrada no curso inferior do rio Negro confere com o demonstrado por Raupp et al. (2009), na investigação sobre as diatomáceas no plâncton do lago Cutiuaú, em comunicação com o rio Jaú na bacia do rio Negro. Maior riqueza de Pinnularia foi também observada no período de águas baixas, devido a maior contribuição de elementos do perifíton e bentos a comunidade do plâncton.

Dentre os 15 táxons específicos e infra-específicos de Pinnularia inventariados no curso inferior do rio Negro, uma espécie ( $P$. sterrenburgii var. sterrenburgii Metzeltin \& Lange-Bertalot) e uma que não a variedade típica ( $P$. subgibba var. capitata Metzeltin \& Krammer) são primeiras citações de ocorrência para a bacia do rio Negro.

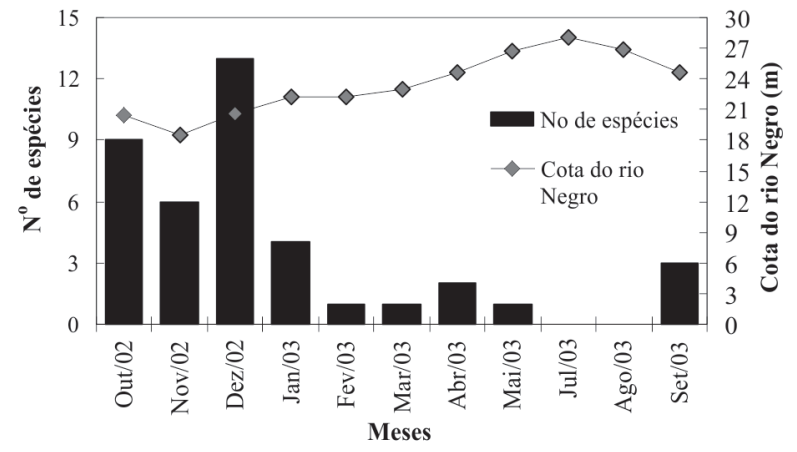

Figura 2 - Variação mensal da riqueza de Pinnularia em relação ao nível hidrométrico do rio Negro, registrado no Porto de Manaus. 
Tabela 2 - Distribuição e frequência (Fr) dos táxons de Pinnularia no curso inferior do rio Negro, no período de outubro de 2002 a setembro de 2003 , ( $E=$ esporádicas, $\mathrm{F}$ = frequentes, $\mathrm{R}=$ raras); (+ presença; - ausência); período ( $\mathrm{B}$ = águas baixas, $\mathrm{E}=$ enchente, $\mathrm{A}=$ águas altas, $\mathrm{V}=$ vazante).

\begin{tabular}{|c|c|c|c|c|c|c|c|c|c|c|c|c|}
\hline \multirow{2}{*}{$\begin{array}{l}\text { Táxons } \\
\text { Meses }\end{array}$} & \multicolumn{3}{|c|}{2002} & \multicolumn{9}{|c|}{2003} \\
\hline & Out & Nov & Dez & Jan & Fev & Mar & Abr & Mai & Jul & Ago & Set & $\mathrm{Fr}$ \\
\hline Período & $A B$ & $A B$ & $A B$ & $E$ & $E$ & $\mathrm{E}$ & $E$ & A & A & V & V & \\
\hline P. aquaenigrae & + & - & + & - & - & + & - & - & - & - & - & $\mathrm{E}$ \\
\hline P. boyeriformis & - & + & + & + & - & - & - & - & - & - & - & $E$ \\
\hline P. confirma & + & + & + & + & - & - & + & + & - & - & + & $\mathrm{F}$ \\
\hline P. instabiliformis & + & - & + & + & - & - & - & - & - & - & + & $E$ \\
\hline P. meridiana var. concava & - & + & + & - & - & - & - & - & - & - & - & $\mathrm{R}$ \\
\hline P. mollenhaueri & + & - & - & - & - & - & - & - & - & - & - & $\mathrm{R}$ \\
\hline P. pogoii & - & - & + & + & - & - & + & - & - & - & - & $\mathrm{E}$ \\
\hline P. perinstabilis & + & - & - & - & - & - & - & - & - & - & - & $\mathrm{R}$ \\
\hline P. permontana & - & + & + & - & - & - & - & - & - & - & - & $\mathrm{R}$ \\
\hline P. perumbrosa & + & + & + & - & - & - & - & - & - & - & - & $E$ \\
\hline P. rhombofasciata & - & - & + & - & - & - & - & - & - & - & - & $\mathrm{R}$ \\
\hline P. rostratissima var. ventricosa & + & + & + & - & + & - & - & - & - & - & - & $\mathrm{E}$ \\
\hline P. sterrenburgii var. sterrenburgii & + & - & + & - & - & - & - & - & - & - & + & $E$ \\
\hline P.subboyeri & - & - & + & - & - & - & - & - & - & - & - & $\mathrm{R}$ \\
\hline P. subgibba var. capitata & + & - & + & - & - & - & - & - & - & - & - & $\mathrm{R}$ \\
\hline Riqueza & 9 & 6 & 13 & 4 & 1 & 1 & 2 & 1 & 0 & 0 & 3 & \\
\hline
\end{tabular}

\section{AGRADECIMENTOS}

Os autores agradecem a Coordenação de Aperfeiçoamento de Pessoal de Nível Superior (CAPES) pela bolsa de doutorado concedida a primeira autora; ao Conselho Nacional de Desenvolvimento Científico e Tecnológico (CNPq) pela bolsa de Produtividade em Pesquisa concedida à segunda autora; pelo auxílio financeiro Proc. 485618/2007-9 e ao INPA/ MCT-CNPq pela bolsa DTI concedida ao terceiro autor, Somos também gratos ao projeto BIOTUPÉ (financiado pelo INPA, CNPq, FAPEAM, SEMMAS, CEULM/ULBRA) que fomentou parte da logística das coletas e de infraestrutura laboratorial.

\section{BIBLIOGRAFIA CITADA}

Bicudo, C.E.M.; Menezes, M. 2006. Genera of continental algae from Brazil: Identification key and descriptions. Rima: São Carlos, São Paulo. 508 pp (in Portuguese).

Diaz-Castro, J.J.; Souza-Mosimann, R.M.; Laudares-Silva, R.; Forsberg, B.R. 2003. Composition of the periphytic diatom community of the Jaú river, Amazonas, Brazil. Acta Amazonica, 33 (4): 583- 606 (in Portuguese, with abstract in English).
Fukushima, H.; Xavier, M.B. 1988. Attached Diatom from the Negoro river, Amazonas, Brazil. Diatom Research, 4:11-16.

Goulding, M.; Barthem, R.; Ferreira, E.J. 2003. The Smithsonian Atlas of Amazon. Princeton Editorial Associates, Oklahoma City, USA. 253 pp.

Hustedt, F. 1965. New and little known diatoms. IX. Freshwater diatoms from Brazil, especially in the Amazon. Internationale Revue der Gesamten hydrobiologie und Hydrographie, 50(3):391410 (in German).

Junk, W.J.; Silva, C.J. 1995. Neotropical floodplains: A comparison between the Pantanal of Mato Grosso and the Large Amazonian river floodplains. p. 195-227. In: J.G. Tundisi; C.E. Bicudo \& T. Matsamura-Tundisi (Eds.). Limnology in Brazil. Brazilian Academy of Science, Brazilian Limnological Society. 376 pp.

Krammer, K. 2000. The Genus Pinnularia. In: Lange-Bertalot, H. (Ed). Diatoms of Europe. Vol. 1. Koeltz Scientific Books. Germany. 703 pp.

Medlin, L.K.; Kaczmarska, I. 2004. Evolution of the diatoms: V. Morphological and cytological support for the major clades and a taxonomic revision. Phycologia, 43(3): 245-270.

Metzeltin, D.; Lange-Bertalot, H. 1998. Tropical Diatoms of the South America I. Iconographia Diatomologica 5. A.R.G. Gantner Verlag K.G. Koenigstein. 695 pp. 
Metzeltin, D.; Lange-Bertalot, H. 2002. Diatoms from the "Island Continent” Madagascar. Iconographia Diatomologica 11. A.R.G. Gantner Verlag K.G. Koenigstein. 286 pp.

Metzeltin, D.; Lange-Bertalot, H. 2007. Tropical Diatoms of the South America II. Iconographia Diatomologica 18: A.R.G. Gantner Verlag K.G. Koenigstein. 877 pp.

Patrick. R.; Reimer, C.W. 1966. The diatoms of United States exclusive of Alaska and Hawaii. Vol. 1, (Monographs.13). Academy of Natural Sciences. Philadelphia. 688 pp.

Pereira, A.C. 2009. Pinnulariaceae (Bacillariophyta) from an Amazonian floodplain lake (Tupé lake, Amazonas, Brazil): taxonomy and seasonal and spatial distribution. Dissertação de Mestrado, Instituto Nacional de Pesquisas da Amazônia, Manaus, Amazonas. 88 pp. (In Portuguese, with abstract in English).

Rai, H.; Hill, G. 1981. Physical and Chemical studies of lago Tupé; a central amazonian black water, "Ria Lake". Internationale Revue der Gesamten hydrobiologie und Hydrographie, 66(1):37-82.

Raupp, S.V.; Torgan, L.C.; Melo, S. 2009. Planktonic diatom composition and abundance in the Amazonian floodplain Cutiuaú Lake are driven by the flood pulse. Acta Limnologica Brasiliensia, 21 (2):227-234.

Round, F.E.; Crawford, R.M.; Mann, D.G. 1990. The diatoms Biology and morphology of the genera. Cambridge University Press, Cambridge. 747 pp.

Schmidt, A. (1874-1959): Atlas of Diatoms. Heft 1-120. Tafeln 1-462 (Tafeln 1 - 216 Schmidt, A.; 217-240 Fricke, F.; 241-244 Heiden, H.; 245, 246 Müller, O.; 247-256 Fricke, F.; 257-264 Heiden, H.; 265-268 Fricke, F; 269-472 Hustedt, F.) 65 Aschersleben-Leipzig (in German).
Simonsen, R. 1987. Atlas and catalogue of the diatom types of Fredrich Hustedt. J. Cramer. Vol. 3. 772 pl. Berlin, Germany.525 pp.

Sioli, H. 1984. The Amazon and its main affluents: Hydrography, morphology of the river courses, and river types, p.127-166. In: Sioli, H. (Ed.). The Amazon: Limnology and Landscape Ecology of a Might Tropical River and its Basin. Dr. W. Junk Publishers., Dordrecht.

Stosch, H. A. von. 1970. Methods for preparation of small or delicate siliceous elements for the electron and light microscopy, especially of diatoms and small quantities of material. Zeitschrift für wissenschaftlichen Mikroscopie, 70: 29-32 (in German).

Uherkovich, G. 1976. Algae from rivers Rio Negro and Rio Tapajós. Amazoniana. 5(4): 465-515 (in German with abstract in Portuguese).

Uherkovich, G. 1984. Phytoplankton, p. 265-310. In: Sioli, H. (Ed.). The Amazon: Limnology and Landscape Ecology of a Might Tropical River and its Basin. Dr. W. Junk Publishers, Dordrecht.

Uherkovich, G.; Rai, H. 1979. Algae from the Rio Negro and its affluents. Amazoniana, 6(4): 611-638 (in German with abstract in Portuguese).

Wetzel, C.E. 2011. Biodiversity and distribution of diatoms (Ochrophyta, Bacillariophyceae) in the basin of Negro river, Amazonas, Brazil. Tese de Doutorado, Instituto de Botânica da Secretaria do Meio Ambiente, São Paulo, São Paulo. 1876 pp. (In Portuguese, with abstract in English).

Recebido em: 27/03/2011

Aceito em: 09/09/2011 
